\title{
Gastric outlet obstruction secondary to percuta- neous endoscopic gastrostomy tube placement
}

We report a complication of percutaneous endoscopic gastrostomy (PEG) insertion in a 60-year-old woman with an endstage neurodegenerative disorder. The first attempt at PEG tube insertion was abandoned due to failure to transilluminate the stomach because of a large hiatus hernia, but a second attempt using a combined fluoroscopic and endoscopic approach was successful. Gastrostomy feeding was commenced 24 hours later following satisfactory postprocedural monitoring. At 48 hours, our patient developed generalized abdominal pain associated with vomiting and fever. Clinical diagnoses of gastrostomy site leakage, gastroparesis, and aspiration pneumonia were made. Subsequent computed tomography (CT) of the abdomen demonstrated the gastrostomy tube traversing the left anterior abdominal wall, "through and through" the gastric antrum, to enter the stomach wall again high on the lesser curvature of the stomach ( $\bullet$ Fig. 1, Fig. 2 , - Fig.3). This transfixed the distal stomach, causing obstruction of the gastric outlet. Attempts to further assess the gastrostomy tube position by esophagogastroscopy failed. The patient's hemoglobin dropped to $5 \mathrm{~g} / \mathrm{dL}$, presumably due to the large volume of blood-stained fluid found in the stomach.

After consideration of the patient's comorbidities we adopted a conservative management involving insertion of a fluoroscopically guided nasojejunal tube for feeding and regular aspiration of the gastrostomy tube for stomach decompression. The long-term plan was to allow the tract to mature and become a controlled gastrocutaneous fistula as described by Milanchi and Wilson [1]. Despite blood transfusions, antibiotics and chest physiotherapy, and nutritional support, our patient died 7 days later.

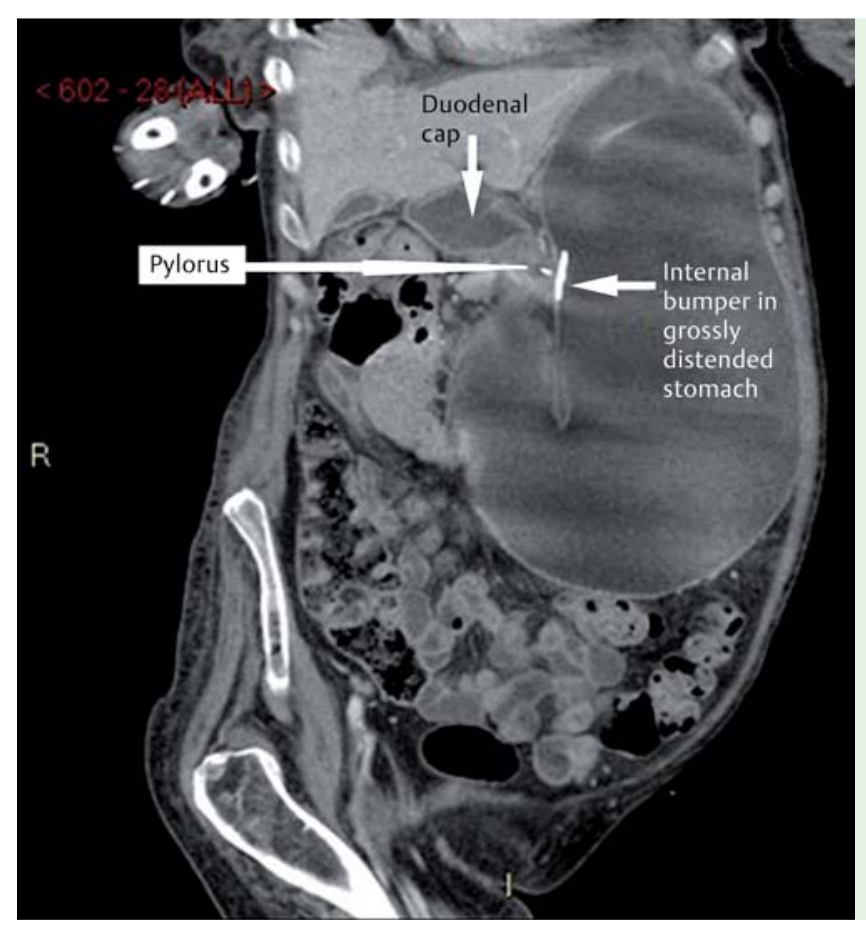

Fig. 1 Oblique sagittal view demonstrating a massively distended stomach. The internal bumper is on the gastric wall adjacent to the pylorus.

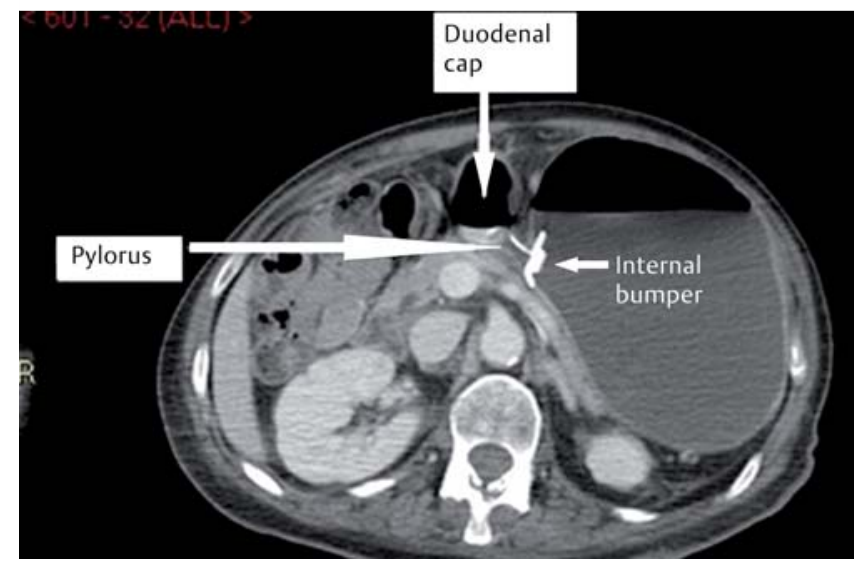

Fig. 2 Axial view showing the internal bumper on the gastric wall; the tube then passes into the pylorus.

Complications after radiologically inserted gastrostomy are more common than after PEG. In this hybrid procedure, a rolling hiatus hernia caused the antrum to get in the way of the needle/PEG tract. CT rather than fluoroscopic guidance might have avoided the complication [3]. Duodenoscopy at the time of PEG tube insertion would have allowed early diagnosis of the impending gastric outlet obstruction.

Endoscopy_UCTN_Code_CPL_1AH_2AI

\section{M. Than ${ }^{1}$, J. Witherspoon ${ }^{1}$, G. Tudor ${ }^{2}$, A. Saklani ${ }^{1}$}

${ }^{1}$ Department of Colorectal Surgery, Princess of Wales Hospital, Bridgend, United Kingdom

${ }^{2}$ Department of Radiology, Princess of Wales Hospital, Bridgend, United Kingdom

Competing interests: None 


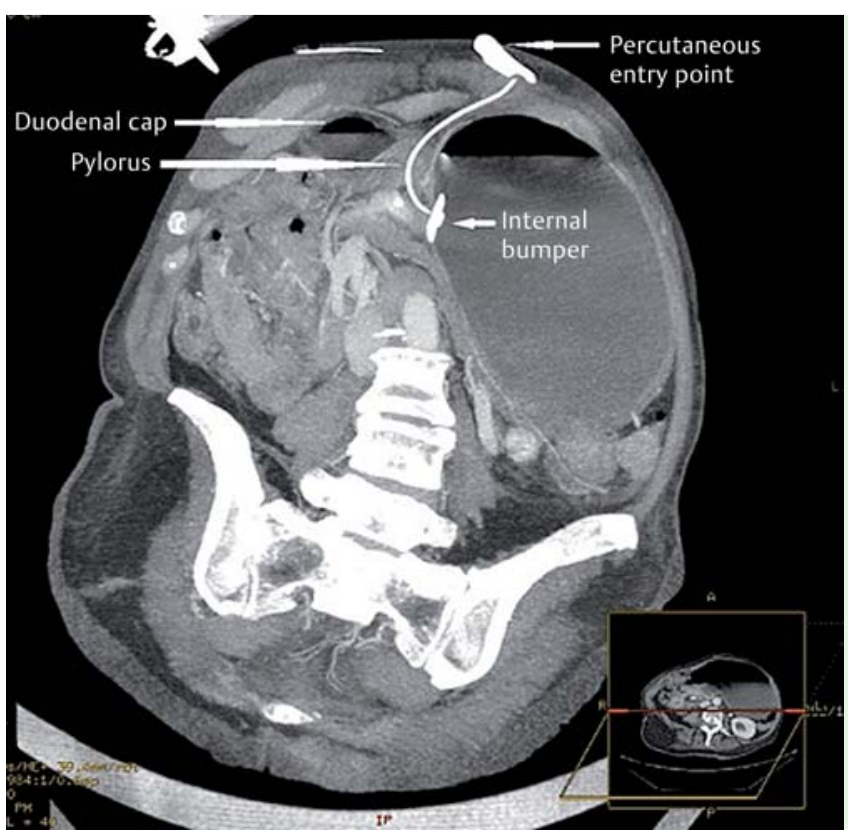

Fig. 3 Course of the

PEG tube.

\section{References}

1 Milanchi S, Wilson MT. Malposition of percutaneous endoscopic-guided gastrostomy: guideline and management. J Minimal Access Surg 2008; 4: 1 -4.

2 Grant DG, Bradley PT, Pothier DD et al. Complications following gastrostomy tube insertion in patients with head and neck cancer: a prospective multi-institution study, sys-
Bibliography

Dol http://dx.doi.org/

10.1055/s-0032-1309712

Endoscopy 2012; 44: E269-E270

(c) Georg Thieme Verlag KG

Stuttgart · New York

ISSN 0013-726X

\section{Corresponding author}

\section{M. Than, MBChB}

Department of Colorectal Surgery Princess of Wales Hospital

Coity Road

Bridgend CF31 1RQ

United Kingdom

Fax: 01656752855

mmthan@doctors.org.uk 